\title{
PREVALENCIA DE LAS CONSECUENCIAS DE CARIES DENTAL NO TRATADA EN ESCOLARES DE 12 AÑOS EN LA PARROQUIA EL VECINO, CUENCA 2016
}

\section{PREVALENCE OF THE CONSEQUENCES OF DENTAL CARIES NOT TREATED IN 12 YEAR OLD SCHOOLS IN THE EL VECINO PARISH, CUENCA 2016}

\author{
Velez Edith $^{1 *}$, Bastidas Zulay ${ }^{2}$. \\ ${ }^{1}$ Odontóloga Rural del Ministerio de Salud de la Repúplica del Ecuador.Ecuador. \\ 2 Docente especialista en Endodoncia de la Universidad Católica de Cuenca.Ecuador. \\ * evveleza36@est.ucacue.edu.ec
}

\begin{abstract}
Resumen
OBJETIVO:El propósito del presente estudio fue determinar la prevalencia de las consecuencias de la caries dental no tratada en escolares de 12 años en la parroquia El Vecino, Cuenca 2016. MATERIALES Y MÉTODOS: Estudio descriptivo, transversal y observacional. Se evaluaron 185 escolares de 12 años, se incluyeron las fichas epidemiológicas de estudiantes matriculados en los centros educativos, se utilizaron los códigos y criterios del índice PUFA para determinar las infecciones odontogénicas como son la pulpitis, úlcera, fístula y absceso. Los examinadores fueron calibrados por un grupo de especialistas y supervisados por un epidemiólogo experimentado (Kappa $>80 \%$ ). Todos los exámenes bucales fueron desarrollados con luz natural y espejo bucal antes del recreo. RESULTADOS: La muestra presentó $8.1 \%$ prevalencia de las consecuencias de caries dental no tratada; de los cuales se presentó $5.4 \%$ en el sexo femenino y $2.7 \%$ sexo masculino, siendo la pulpitis la principal infección odontogénica presente en 18 piezas dentales dando un índice PUFA $9.7 \%$, además $61.1 \%$ fueron los primeros molares superiores afectados y un $38.9 \%$ primeros molares inferiores, siendo el primer molar superior izquierdo el más frecuente $33 \%$. CONCLUSIONES: Esta investigación reveló, que la prevalencia de las consecuencias de la caries dental no tratada fue $8.1 \%$, mientras que la principal afección fue la pulpitis con un índice PUFA $9.7 \%$.
\end{abstract}

Palabras clave: Absceso, Caries dental, Epidemiología, Fístula dental, Pulpitis, Úlceras bucales.

\begin{abstract}
OBJETIVE:The purpose of this study was to determine the prevalence of dental caries result of untreated schoolchildren 12 years in the parish El Vecino, Cuenca 2016. MATERIAL AND METHODS: Descriptive, transverse and observational study. A total of 185 schoolchildren aged 12 years, epidemiological records of students enrolled in educational centers were evaluated, PUFA index codes and criteria were used to determine odontogenic infections such as pulpitis, ulcer, fistula and abscess. The examiners were calibrated by a group of specialists and supervised by an experienced epidemiologist (Kappa> 80\%). All oral exams were developed with natural light and mouth mirror before recess. RESULTS: The sample presented $8.1 \%$ prevalence of the consequences of untreated dental caries giving us a Chi square $p=0.58$; of which 5.4\% were found in females and $2.7 \%$ in males. Pulpitis was the main odontogenic infection present in 18 dental specimens, giving a PUFA index of $9.7 \%$. In addition, $61.1 \%$ were the first impacted upper molars and 38.9\% Lower molars, with the upper left first molar being the most frequent 33\%. CONCLUSIONS: This investigation revealed that the prevalence of the consequences of untreated dental caries was $8.1 \%$, while the primary condition with pulp with a PUFA $9.7 \%$.
\end{abstract}

Key words: Abscess, Dental Caries, Epidemiology, Dental fistula, Pulpitis, Oral Ulcer.

\section{INTRODUCCIÓN}

Según la Organización Mundial de la Salud, (OMS) en su último estudio, de Salud Bucodental en América Latina se estima que aproximadamente del $60 \%$ a $90 \%$ de los escola- res presentan caries dental, siendo la causa más importante de la pérdida de las piezas dentales en las personas jóvenes. ${ }^{1-3}$

"La caries dental es una de las enfermedades de mayor prevalencia en el mundo" ${ }^{4,5}$ y pese a que existen numerosos 
estudios y avances científicos en la actualidad no se ha logrado erradicarla, algunos de los detonantes para que se produzca esta patología, son los programas de prevención de enfermedades odontológicas insuficientes, la mala técnica de higiene oral, entre otros. Dando como resultado que esta enfermedad genere altos costos para tratarla, además de disminuir las funciones masticatorias hasta llegar a eliminar los alimentos duros de la dieta debido a la presencia de dolor y malestar causados por los problemas orales como son la caries dental, por lo tanto esta patología es una enfermedad que no solo perturba la salud bucal, también puede llegar a afectar la calidad de vida del individuo y de quienes lo rodean, convirtiéndose en un problema de salud pública. ${ }^{5,6}$

En vista de la epidemia mundial de caries dental no tratada en niños, y de los pocos reportes respecto a la situación de salud bucal en la sociedad, fue motivo necesario para realizar un estudio descriptivo epidemiológico, con la finalidad de saber la magnitud del problema de salud bucal en la ciudad de Cuenca.

La mayoría de los estudios epidemiológicos realizados ampliamente en todo el mundo, según lo descrito por Organización Mundial de la Salud, (OMS) utilizan el índice CPO-D / ceod para evaluar la experiencia de caries $^{6-9}$ Una desventaja de este índice es que no consigue registrar, las lesiones causadas por las consecuencias de la caries dental no tratada como pulpitis, úlceras, fístulas o abscesos.

Dada la magnitud de esta necesidad en el año 2010, Monse et al, introdujo el índice PUFA/pufa. Este índice complementa a los índices de caries existentes, mediante la visualización de la gravedad de la caries dental y la cuantificación de infecciones odontogénicas de la pulpa y los tejidos circundantes, debido a la consecuencia de caries dental sin tratar, tanto en la dentición temporal (pufa) como en la dentición permanente (PUFA). Este índice es universalmente aplicable en todos los escenarios, incluyendo las condiciones de campo simple.

Por esta razón, el propósito del presente estudio fue evaluar y determinar la frecuencia de las consecuencias de caries dental no tratadas en escolares de 12 años de la parroquia El Vecino.

\section{MATERIALES Y MÉTODOS}

La metodología aplicada fue descriptiva, transversal y observacional. La población fue de 185 fichas de escolares de 12 años de edad, esta cantidad de fichas proviene de una población de 675 escolares matriculados en las Unidades Educativas pertenecientes a la parroquia El Vecino, para lo cual se aplicó la fórmula de muestreo. Los valores fueron recolectados de una base de datos de la Universidad Católica de Cuenca, Facultad de Odontología los cuales constaban en un programa OPEN EPI 7.2. De la población estudiada se incluyeron, las fichas epidemiológicas de estudiantes matriculados en los centros educativos, que tengan 12 años de edad cumplidos o que vayan a cumplir hasta el 31 de diciembre del 2016, que consten en dicha bases de datos además de que cada ficha tenga el consentimiento informado de los padres de familia o apoderado y también que den los escolares su propio asentimiento, y se excluyeron del estudio las fichas con incoherencias y que no se entiendan los datos. La base de datos fue exportada a un archivo de Excel y se verificó su exactitud. Para la obtención de las fichas registradas en la base de datos, los examinadores escogieron al azar diferentes unidades educativas fiscales pertenecientes a uno de los sectores urbanos más populares de la ciudad de Cuenca, y localizada en la parte norte del centro histórico de la ciudad, la muestra se obtuvo de las Unidades Educativas como son la "Escuela de educación básica Mary Coryle", "Escuela Luis Roberto Bravo", "República de Chile", "Unidad Educativa Juan Montalvo" y la "Unidad Educativa Zoila Esperanza Palacio". Los examinadores fueron calibrados por un grupo de especialistas y supervisados por un epidemiólogo experimentado (Kappa $>80 \%$ ). Todos los exámenes bucales fueron desarrollados con luz natural y espejo bucal antes del recreo.

Este estudio buscó describir cuantitativamente el problema en personas de 12 años de edad, utilizando los parámetros descritos por Monse et al, para registrar la presencia de las consecuencias de caries dental no tratada ${ }^{6-15}$ que se detalla a continuación. Criterios y códigos del índice PUFA / pufa:

P / p: Pulpitis: Significa afección de la pulpa y se registra cuando el proceso de la caries dental, está avanzado que la apertura de la cámara de la pulpa es visible, o cuando la estructura coronal del diente ha sido destruida por el proceso de caries y sólo quedan las raíces. Además, su diagnóstico es sin sonda. (Figura 1).

U / u: Ulceración: Es una ulceración de los tejidos blandos causados por bordes afilados de un diente dislocado o fracturado con afección de la pulpa o fragmentos de raíz han provocado ulceración traumática de los tejidos blandos circundantes, por ejemplo, la lengua o la mucosa bucal. (Figura 1).

F / f: Fístula: Es un contenido purulento producido por la descomposición del tejido pulpar, que atraviesa el tracto sinusal, terminando en la mucosa bucal, en relación con la pieza dental con compromiso pulpar.

A / a: Absceso: Se registra cuando se observa un contenido purulento, debido a una infección asociada a un diente con compromiso pulpar, pero sin comunicación al medio bucal, es decir sin presencia de un tracto sinusal. (Figura 1).

Como examinar el índice PUFA/pufa:

La puntuación del índice PUFA/pufa por persona se calcula de la misma manera que el índice CPOD/ceod.

Se le asigna un código por diente, en caso de duda sobre el alcance de la infección odontogénica, se codifica con la letra $(\mathrm{P} / \mathrm{p})$ pulpitis. Además la evaluación se realiza visualmente sin el uso de un instrumento. Si el diente deciduo y su diente sucesor permanente están presentes y los dos se ven involucrados en la infección odontogénica, se registra por 

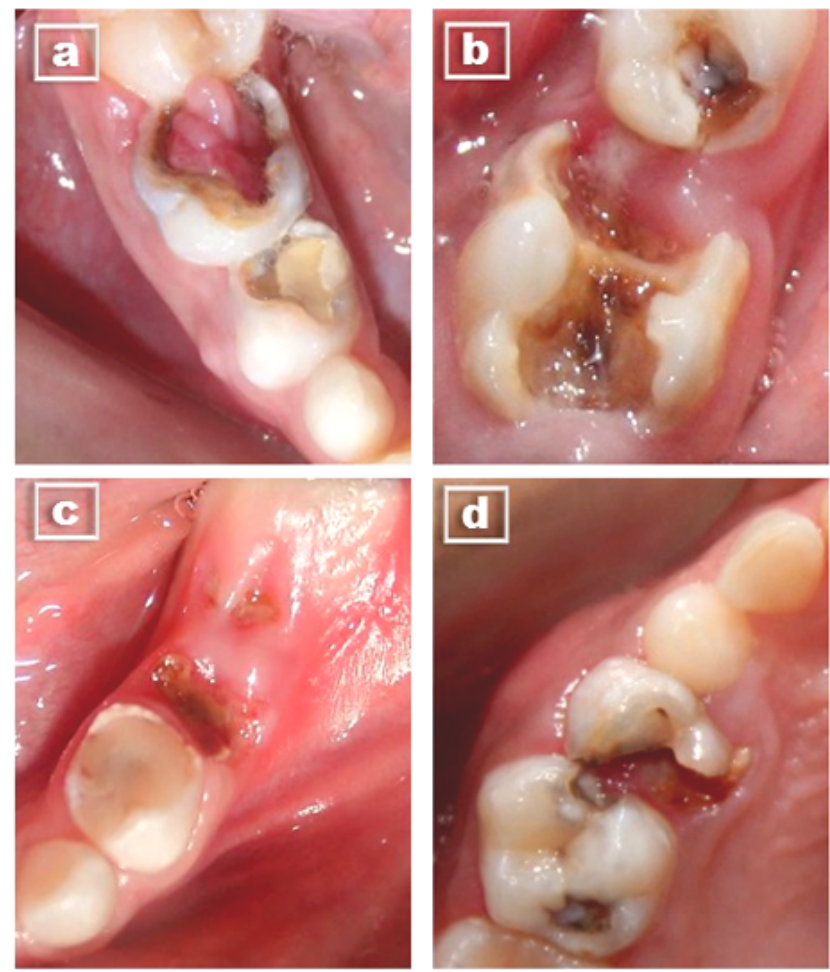

Fig. 1. (a-b) Pulpitis- compromiso pulpar visible (P/p). (b) Ulceración causada por fragmentos remanentes cortantes (U/u), (c) Absceso - es una inflamación que contiene pus asociada a un diente con pulpitis (A/a). Fuente: Pariona-Minaya M. (2016). ${ }^{21}$

separado.

Se usaron las letras mayúsculas para dentición permanente (PUFA) y minúsculas para dentición temporal (pufa). ${ }^{16}$ Además el índice PUFA/pufa para los dientes permanentes y temporales se registra por separado. La puntuación puede variar desde 0 a 20 pufa para la dentición temporal y 0-32 PUFA para la dentición permanente.

\section{RESULTADOS}

Se evaluaron un total de 185 fichas epidemiológicas con un rango de edad de escolares de 12 años, se obtuvo una muestra de los cuales $91.9 \%(\mathrm{n}=170)$ fueron escolares sanos y en un $8.1 \%(n=15)$ de los escolares se presentaron enfermos a causa de las consecuencias de caries dental no tratada principalmente por pulpitis en 18 piezas dentales afectadas obteniendo un índice PUFA del $9.7 \%(n=18)$. (Tabla $1-3)$.

En el $5.4 \%(n=10)$ de los escolares del sexo femenino se observaron infecciones odontogénicas, mientras que en los estudiantes del sexo masculino presentaron un $2.7 \%(n=5)$ de infecciones, por lo cual no existe una diferencia significativa. (Tabla 2).

Además, señala que las piezas con mayor prevalencia de afectación son los primeros molares superiores con un $61.1 \%$ $(n=11)$ y los primeros molares inferiores en un $38.9 \%(n=7)$,

\begin{tabular}{lrr}
\hline & $\mathrm{n}$ & $\%$ \\
\hline Con Consecuencias & 15 & 8.1 \\
Sin Consecuencias & 170 & 91.9 \\
Total & 185 & 100.0 \\
\hline
\end{tabular}

Tabla 1. Prevalencia de la consecuencia de caries dental no tratada en escolares de 12 años de la parroquia El Vecino, Cuenca 2016.

\begin{tabular}{lrrrrrrr}
\hline \multirow{2}{*}{ CONS. CARIES } & \multicolumn{9}{c}{ FEMENINO } & \multicolumn{2}{c}{ MASCULINO } & \multicolumn{2}{c}{ TOTAL } \\
& $\mathbf{n}$ & $\%$ & $\mathbf{n}$ & $\%$ & $\mathbf{n}$ & $\%$ \\
\hline Con Consecuencia & 10 & 5.4 & 5 & 2.7 & 15 & 8.1 \\
Sin Consecuencia & 101 & 54.6 & 69 & 37.3 & 170 & 91.9 \\
Total & 111 & 60.0 & 74 & 40.0 & 185 & 100.0 \\
\hline & & \multicolumn{5}{c}{ Chi cuadrado $p=0.58$}
\end{tabular}

Tabla 2. Distribución de la muestra de acuerdo al sexo en los escolares de 12 años de edad de la parroquia El Vecino, Cuenca 2016.

siendo el primer molar superior izquierdo el más frecuente con un $33 \%(n=6)$. Ver Tabla 4 - Grf. 1.

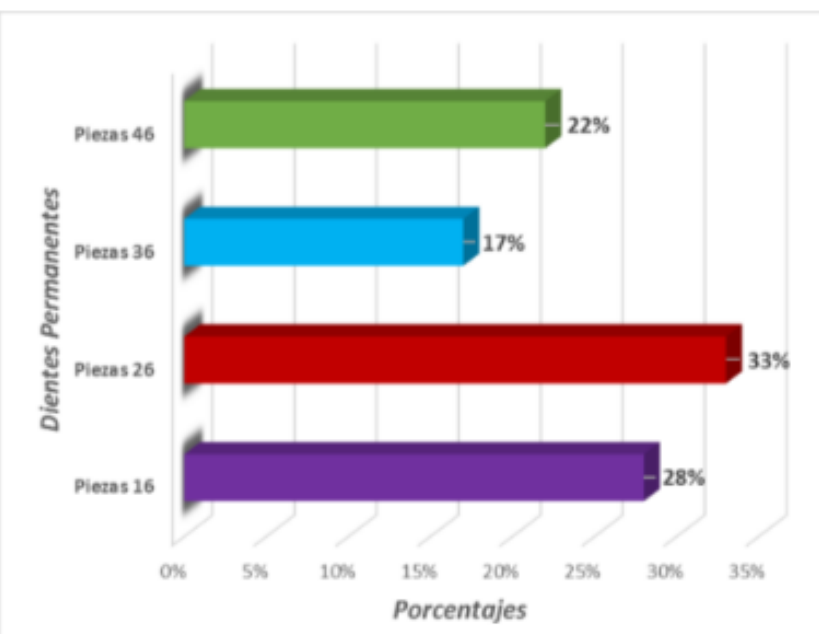

Grf. 1. Distribución del diente permanente más afectado. 


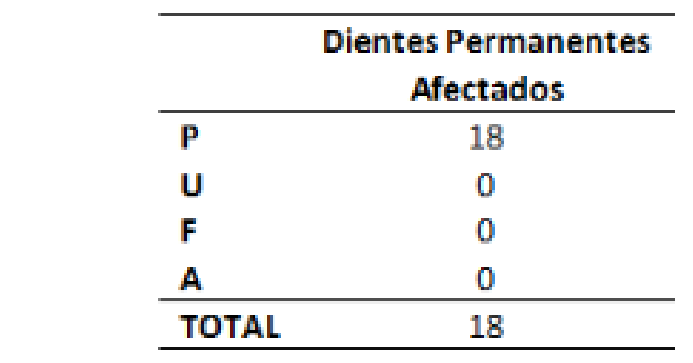

Îndice PUFA

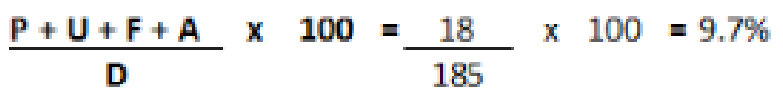

Tabla 3. Prevalencia del índice PUFA.

\begin{tabular}{lrr}
\hline & $\mathbf{n}$ & \% \\
\hline Primeros Molares Superiores & 11 & 61.1 \\
Primeros Molares Inferiores & 7 & 38.9 \\
TOTAL & 18 & 100.0 \\
\hline
\end{tabular}

Tabla 4. Distribución del diente permanente más afectado.

\section{DISCUSIÓN}

Los estudios sobre la epidemiología de la caries dental en los últimos años, se han centrado en crear más criterios de diagnóstico para permitir la evaluación de las etapas iniciales de caries dental. ${ }^{6,7}$ Sin embargo, la Organización Mundial de la Salud (OMS) aún utiliza índices tradicionales como son los índices CPOD / ceod que permiten evaluar el estado del proceso carioso, por lo tanto el índice PUFA va complementar a los índices antes mencionados, registrando la prevalencia de las consecuencias de caries dental no tratadas, que no solo afecta la cavidad bucal sino también la salud general y la calidad de vida de los niños.

En esta investigación la población de estudio fue de 185 fichas epidemiológicas en escolares de 12 años de edad de la parroquia El Vecino, donde la prevalencia de las consecuencias de las caries dental no tratada se encontró en un $8.1 \%$ de los niños que presentaban infecciones odontogénicas, este resultado es menor en comparación al levantamiento epidemiológico Nacional realizado en Filipinas por Monse et al, ${ }^{6}$ (56\% en niños 12 años), Benzian et al, ${ }^{7} 55.7 \%$, Shanbhog et al, $^{8} 37,7 \%$, Ramazani et al ${ }^{9} 30 \%$, Oziegbe et al, ${ }^{10} 9,2 \%$, Jain et al, ${ }^{11}$ registraron $0.3 \%$, Jazrawi et al, ${ }^{12} 0,12 \%$, Murthy et al, ${ }^{13}$ (19.4\% en escolares 12 a 15 años), Estos valores difieren de nuestra investigación, debido a los hábitos alimenticios, experiencias pasadas, falta de un plan escolar de prevención, falta de higiene oral, falta de servicios de atención dental, y negligencia por parte de los profesionales de la salud. ${ }^{13,17,18}$ Mientras que estudios realizados en escolares Brasileños por Figueiredo et al, ${ }^{14}$ y Leal et al, ${ }^{15}$ de 6 y 7 años fué $23,7 \%$ y $26,2 \%$ respectivamente, y de 8 a 10 años por Mota et al, ${ }^{16} 17,9 \%$, Baginska et al, ${ }^{17}(43 \%, 72,4 \%$ entre $5-7$ años), Mehta et al, ${ }^{18} 38,6 \%$, y Tanvi et al, ${ }^{19} 61 \%$, en estas investigaciones el porcentaje es más elevado que los valores de nuestro estudio, puesto que se realizó en edades donde la prevalencia de la consecuencia de caries dental no tratadas se presentan en dentición mixta. ${ }^{1}$

Referente a la prevalencia de las consecuencias de caries dental no tratadas de acuerdo al sexo, el estudio mostró que existía mayor incidencia en el sexo femenino $5.4 \%$ que del sexo masculino $2.7 \%$ se creé que es debido a la erupción temprana de los dientes en las mujeres. En una investigación hecha por Murthy et al, ${ }^{13}$ se encontró que había mayor prevalencia en el sexo masculino con $62 \%$ y en el sexo femenino $53 \%$ no habiendo diferencia significativa en la muestra.

Respecto a la frecuencia de las consecuencias de caries dental no tratada en nuestra investigación fue de 18 piezas dentales afectadas obteniendo un $9.7 \%$ del índice PUFA siendo la pulpitis, la afección odontogénica más frecuente, este porcentaje es menor, a diferencia de los estudios realizados en escolares entre 12 a 15 años de edad en Filipinas $41 \%,{ }^{6}$ Brasil $19.5 \%,{ }^{14}$ y la India $80.6 \%,{ }^{16} 31.1 \%$, que nos muestran un porcentaje mayor de afección odontogénicas a nivel pulpar, debido a que sus muestras de estudio fueron a nivel nacional.

Una investigación realizada en la ciudad de Ventanilla, Perú en el año 2014 por Pachas et al, ${ }^{20}$ respecto a la frecuencia del índice PUFA fue de $1.29 \%$ de las piezas evaluadas, el cual representa un valor menor al obtenido en nuestro estudio, debido que la muestra de estudio fue realizada en dentición mixta.

En este trabajo se analizaron 5180 piezas dentales de una población de 185 escolares de 12 años de edad, observando un $61.1 \%$ de los primeros molares superiores afectados y en un $38.9 \%$ los primeros molares inferiores se encontraron con infecciones odontogénicas, siendo el primer molar superior izquierdo el más afectado con un $33 \%$. Debido a su morfología oclusal, presencia y acumulación de placa bacteriana y a su cronología de erupción se considera el diente permanente más susceptible.

\section{CONCLUSIÓN.}

Esta investigación reveló, que la prevalencia de las consecuencias de la caries dental no tratada fue $8.1 \%$, mientras que principal afección fue la pulpitis con un índice PUFA 9.7.

\section{RECOMENDACIONES}

Se recomienda seguir realizando estudios y evaluando las distintas poblaciones, desde aquellas donde se están implementando políticas públicas de salud bucal hasta otras que 
han estado históricamente desatendidas. Esto permitirá crear 1 una visión general, de cuanto afecta las consecuencias de caries dental no tratadas en la calidad de vida, en especial en los pacientes pediátricos, que se los considera un grupo prioritario en la atención comunitaria, ya que ellos tienen un alto riesgo para las caries, mal oclusión, trauma dental, alteraciones en tejidos blandos, e incluso porque están en etapa de crecimiento y desarrollo, con constantes cambios en el ambiente bucal.

\section{CONFLICTO DE INTERESES Y FINANCIAMIEN- TO}

Los autores declaran no tener conflicto de intereses, haber cumplido con los requisitos de autoría y haber autofinanciado este artículo.

\section{Referencias}

1 Ministerio de Salud Pública. Protocolos Odontológicos. Salud Bucal. Primera ed. Dirección Nacional de Normalización. Quito: MINSA. 2015.

2 Abanto J, Bonecker M, Prócida D. Impacto de los problemas bucales sobre la calidad de vida de los niños. Rev. Est. Her. 2010; 20(1): 38-43.

3 Mehta A. Comprehensive review of caries assessment systems developed over the last decade. RSBO. 2012; 9(3): 316-321.

4 Velez E, Bastidas Z. Prevalencia de las consecuencias de caries dental no tratada en escolares de 12 años en la parroquia El Vecino, Cuenca 2016. Ciudad de Cuenca. Universidad Católica de Cuenca 2016.

5 Martins S, Alvarez E, Abanto J, Cabrera A, Lopez R, Masoli $\mathrm{C}$, y cols. Epidemiología de la caries dental en américa Latina. ALOP. 2014; 4 (2).

6 Monse B., Heinrich-Weltzien R, Benzian H, Holmgren C, van Palenstein Helderman W. PUFA - An index of clinical consequences of untreated dental caries. Community Dent Oral Epidemiol. 2010; 38(1): 77-82.

7 Benzian H, Monse B, Heinrich-Weltzien R, Hobdell M, Mulder J, van Palenstein Helderman W. Untreated severe dental decay: a neglected determinant of low Body Mass Index in 12-year-old Filipino children. BMC Public Health. 2011; 11(558): 1-9.

8 Shanbhog R, Godhi B, Nandlal B, Kumar S, Raju V, Rashmi S. Clinical consequences of untreated dental caries evaluated using PUFA index in orphanage children from India. Int J Oral Health. 2013; 5(5):1-9.

9 Ramazani N, Rezaei S. Evaluation of the Prevalence of Clinical Consequences of Untreated Dental Caries Using PUFA/pufa Index in a Group of Iranian Children. Iranian Journal of Pediatrics. 2016; 1-6.

10 Oziegbe E, Esan T. Prevalence and clinical consequences of untreated dental caries using PUFA index in suburban Nigerian school children. Eur Arch Paediatr Dent. 2013; 14(4):227-231.
1 Jain K, Singh B, Dubey A, Avinash A. Clinical Assessment of Effects of Untreated Dental Caries in School Going Children Using PUFA Index. Chettinad Health City Medical Journal. 2014; 3(3): 105 - 108.

12 Jazrawi K. Evaluation of the Sequelae of Untreated Dental Caries Using PUFA Index. Al- Rafidain Dent J. 2014; 14(1): 101-110.

3 Murthy A, Pramila M, Ranganath S. Prevalence of clinical consequences of untreated dental caries and its relation to dental fear among 12-15-year-old schoolchildren in Bangalore city, India. Eur Arch Paediatr Dent. 2014; 15 (1):45-49.

14 Figueiredo M, Amorim R, Leal S, Mulder J, Frencken J. Prevalence and Severity of Clinical Consequences of Untreated Dentine Carious Lesions in Children from a Deprived Area of Brazil. Caries Res. 2011; 45 (5): 435-442.

15 Leal S. Bronkhorst E, Fan M, Frencken J. Untreated cavitated dentine lesions: Impact on children's quality of life. Caries Res. 2012; 46 (2): 102-106.

16 Mota I, Soares M, Mota B, Silva L, Ramos M, Ramos J. Impact of untreated dental caries and its clinical consequences on the oral health-related quality of life of schoolchildren aged 8-10 years. Qual Life Res. 2016; 25 (1):193-199.

17 Bagińska J, Rodakowska E, Wilczynska-Borawska M, Jamiolkowski J. Index of clinical consequences of untreated dental caries (pufa) in primary dentition of children from north-east Poland. Adv Med Sci. 2013; 58(2):442-447.

18 Mehta A, Bhalla S. Assessing consequences of untreated carious lesions using pufa index among 5-6 years old school children in an urban Indian population. Indian J Dent Res. 2014; 25 (2): 150-153.

19 Tanvi P, Nagar P, Borse M, P J. Untreated severe dental decay- A neglected determinant of child's oral health. Int J Comp Med Res. 2016. 2343 - 2345.

20 Pachas F, Garcia C, Carrasco M, Manrique J, Orejuela F, Cordova D, et al. Diagnóstico epidemiológico sobre caries dental y consecuencias clínicas en escolares de Ventanilla 2014. Mast Chall Fac. Estom UPCH. 2015.

21 Herrera, Dayana, Apaza, Frank, Pariona, Maria, Y Vilca, Laura. "Necesidad de tratamiento endodóntico en escolares de seis años en dos parroquias de la ciudad de Cuenca 2016.. ${ }^{\circ}$ dontología Activa.2016. Volumen 1 Número 2.

Recibido: 16 de enero de 2017. Aceptado: 01 de diciembre de 2017. 
